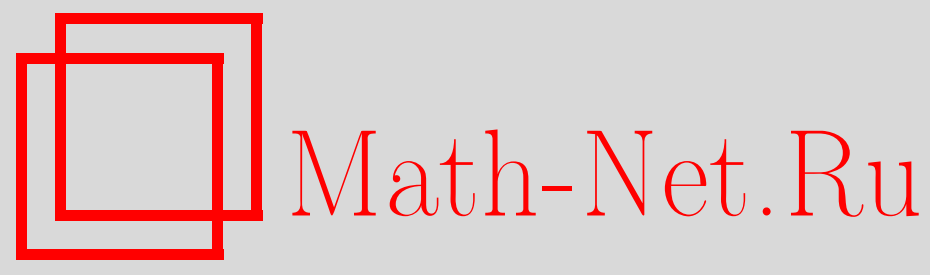

В. М. Петроградский, О функциях сложности для $T$ идеалов ассоциативных алгебр, Матем. заметки, 2000, том 68, выпуск 6, 887-897

DOI: https://doi.org/10.4213/mzm1012

Использование Общероссийского математического портала Math-Net.Ru подразумевает, что вы прочитали и согласны с пользовательским соглашением http://www . mathnet.ru/rus/agreement

Параметры загрузки:

IP: 54.166 .219 .16

26 апреля 2023 г., 13:55:12

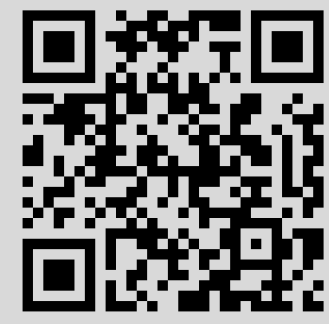




\section{О ФУНКЦИЯХ СЛОЖНОСТИ ДЛЯ $Т$-ИДЕАЛОВ АССОЦИАТИВНЫХ АЛГЕБР}

\section{В.М. Петроградский}

Пусть $c_{n}(\mathbf{V})$ - последовательность роста коразмерностей для многообразия ассоциативных алгебр $\mathbf{V}$. Изучается функция сложности $\mathscr{C}(\mathbf{V}, z)=\sum_{n=0}^{\infty} c_{n}(\mathbf{V}) z^{n} / n$ !. Это экспоненциальная производящая функция для последовательности коразмерностей. Ранее функции сложности использовались для изучения многообразий алгебр Ли. Цель заметки - начать систематическое изучение функций сложности в ассоциативном случае. Эти функции оказываются удобным инструментом для изучения роста многообразий над полем произвольной характеристики. В настоящей заметке найдена формула Шрайера для функций сложности односторонних идеалов свободной ассоциативной алгебры. Она применена для изучения произведений $T$-идеалов. Получена точная формула функции сложности для многообразия $\mathbf{U}_{c}$ ассоциативных алгебр, порожденного алгеброй верхнетреугольных матриц. Доказано, что функция $c_{n}\left(\mathbf{U}_{c}\right)$ является квазиполиномом. Изучаются функции сложности для собственных тождеств. Результаты о функциях сложности применены для изучения асимптотики роста коразмерностей. Прослеживаются аналогии между функциями сложности многообразий и рядами Гильберта-Пуанкаре конечно порожденных алгебр.

Библиография: 20 названий.

1. Введение: рост коразмерностей и функции сложности. Пусть $K$ обозначает основное поле, которое, если специально не оговорено, считаем произвольным. Рассмотрим многообразие (ассоциативных) алгебр V, т. е. класс всех алгебр, удовлетворяющих некоторому множеству тождественных соотношений. Пусть $F(\mathbf{V}, X)$ - его свободная алгебра, порожденная счетным множеством свободных порождающих $X=$ $\left\{x_{i} \mid i \in \mathbb{N}\right\}$. Обозначим через $P_{n}(\mathbf{V}) \subset F(\mathbf{V}, X)$ подпространство всех полилинейных элементов степени $n$ на множестве $\left\{x_{1}, \ldots, x_{n}\right\}$ и рассмотрим размерность этого подпространства

$$
c_{n}(\mathbf{V})=c_{n}(F(\mathbf{V}, X), X)=\operatorname{dim}_{K} P_{n}(\mathbf{V}), \quad n=0,1,2, \ldots
$$

Возникает последовательность коразмерностей $c_{n}(\mathbf{V}), n=0,1,2, \ldots$, которая является важной характеристикой многообразия $\mathbf{V}$. Многообразие, заданное тождествами алгебры $B$, обозначаем $\operatorname{var} B$; через $T(\mathbf{V})$ обозначаем идеал тождеств многообразия $\mathbf{V}$.

Работа выполнена при частичной финансовой поддержке Российского фонда фундаментальых исследований, грант № 98-01-01-20, программы “Университеты России”, грант № 1357, и гранта Минобразования России. 
В случае нетривиального многообразия ассоциативных алгебр последовательность $c_{n}(\mathbf{V})$ ограничена некоторой экспонентой; этот факт был существенно использован Регевым для доказательства того, что тензорное произведение РІ-алгебр всегда является PI-алгеброй [1]. Размыслов предложил рассматривать в случае многообразий алгебр Ли $\mathbf{V}$ функиию сложсности

$$
\mathscr{C}(\mathbf{V}, z)=\sum_{n=0}^{\infty} \frac{c_{n}(\mathbf{V})}{n !} z^{n}, \quad z \in \mathbb{C} .
$$

Если $\mathbf{V}$ - нетривиальное многообразие алгебр Ли, то $\mathscr{C}(\mathbf{V}, z)$ является целой функцией комплексного переменного [2]. В отличие от ассоциативного случая, рост в этом случае может быть сверхэкспоненциальным [3]. Применение функций сложности для многообразий алгебр Ли оказалось плодотворньм и привело к классификации типов роста $[4],[5]$.

Главньм образом, результаты о росте ассоциативных многообразий относятся к полю характеристики нуль, например известны точные асимптотики для так называемых первичных многообразий [6]. Основным подходом при этом является использование теории представлений симметрической групшы. О росте же над полем положительной характеристики известно мало.

Функции сложности для изучения роста ассоциативных многообразий фактически не применялись. Цель заметки - начать систематическое изучение функций сложности в ассоциативном случае. Эти функции оказываются удобным инструментом для изучения роста многообразий над полем произвольной характеристики. Основные результаты работы состоят в следующем. Найдена формула Шрайера для функций сложности односторонних идеалов свободной ассоциативной алгебры (теорема 2.1); она применяется для изучения произведений $T$-идеалов. В качестве приложения получена точная формула функции сложности для многообразия ассоциативных алгебр $\mathbf{U}_{c}$, порожденного алгеброй верхнетреугольных матриц над произвольным полем (теорема 3.1). Это позволило доказать, что функция $c_{n}\left(\mathbf{U}_{c}\right), n=0,1,2, \ldots$, является квазиполиномом. Изучаются функции сложности для собственных тождеств. Показано приложение функций сложности для изучения асимптотики роста коразмерностей. На протяжении всей заметки прослеживаются четкие аналогии между функциями сложности многообразий и рядами Гильберта-Пуанкаре для конечнопорожденных алгебр.

Напомним сначала основные свойства функций сложности. Функция сложности это один из примеров әкспоненциальных производящих функиий, которые широко используются в комбинаторике [7]. Мы будем их рассматривать в несколько более общей ситуации, а именно для подмножеств счетно порожденной алгебры (группоида).

Пусть дана алгебра (группоид), порожденная множеством $X=\left\{x_{i} \mid i \in \mathbb{N}\right\}$. Пусть $A$ - подпространство (подмножество) в этой алгебре. Для любого множества различных элементов $\widetilde{X}=\left\{x_{i_{1}}, \ldots, x_{i_{n}}\right\} \subset X$ мы обозначим через $P_{n}(A, \widetilde{X})$ линейную оболочку (подмножество) всех полилинейных элементов степени $n$ из элементов $\widetilde{X}$ в $A$. Допустим, что размерность этой линейной оболочки (число элементов) $c_{n}(A, \widetilde{X})$ не зависит от $\widetilde{X}$, а зависит только от $n$. В этом случае мы пишем $c_{n}(A)=c_{n}(A, \widetilde{X})$ и говорим, что $A$ является $X$-равномерным, или мы говорим, что для $A$ определена функиия сложсности по отношению $\kappa X$, которая записывается так:

$$
\mathscr{C}(A, z)=\mathscr{C}_{X}(A, z)=\sum_{n=0}^{\infty} \frac{c_{n}(A)}{n !} z^{n}, \quad z \in \mathbb{C} .
$$


Отметим, что $A$ не обязательно должно состоять из однородньх элементов.

Следующая лемма объясняет применение функций сложности [7], [4] и [5].

ЛЕмма 1.1 [5]. Пусть Г - әруппоид (алгебра), порожденная множеством $X=$ $\left\{x_{i} \mid i \in \mathbb{N}\right\}$, с умножением, обозначаемым *. Предположим, что подмножества (подпространства) $A \subset \Gamma, B \subset \Gamma$ являются $X$-равномерными.

1) Пусть $C=A \cup B$, причем пересечение пусто $(C=A \oplus B-$ прямая сумма). Тогда $C$ является $X$-равномерным и $\mathscr{C}(C, z)=\mathscr{C}(A, z)+\mathscr{C}(B, z)$.

2) Допустим что все полилинейные әлементы мнохсества $a * b, a \in A, b \in B$, различны (все полилинейные әлементы среди $\left\{a_{i} * b_{j} \mid i \in I, j \in J\right\}$ линейно независимы при условии, что взяты линейно независимые множества $\left.\left\{a_{i} \mid i \in I\right\} \subset A u\left\{b_{j} \mid j \in J\right\} \subset B\right)$. Тогда $A * B$ также $X$-равномерно, причем $\mathscr{C}(A * B, z)=\mathscr{C}(A, z) \cdot \mathscr{C}(B, z)$.

Пусть $f(z)$ - целая функция. Обозначим $M_{f}(r)=\max _{|z|=r}|f(z)|$. Если $M_{f}(r) \leqslant$ $\exp (\mu r)$ при достаточно больших $r>0$, то говорят, что $f(z)$ әкспоненииального типа. По теореме Регева [1] таковы функции сложности для ассоциативных многообразий. По определению экспоненты $\exp (\alpha z)=\sum_{n=0}^{\infty} \alpha^{n} z^{n} / n$ ! аналогично получается следующий факт.

ЛЕмМА 1.2 [8]. Пусть

$$
f(z)=\sum_{n=0}^{\infty} \frac{a_{n}}{n !} z^{n}
$$

- иелая функиия әкспоненииального типа. Тогда

$$
\varlimsup_{n \rightarrow \infty} \sqrt[n]{\left|a_{n}\right|}=\varlimsup_{r \rightarrow \infty} \frac{\ln M_{f}(r)}{r} .
$$

Определим (верхнюю) әкспоненту и нижнюю әкспоненту многообразия формулами

$$
\operatorname{Exp} \mathbf{V}=\varlimsup_{n \rightarrow \infty} \sqrt[n]{c_{n}(\mathbf{V})}, \quad \underline{\operatorname{Exp}} \mathbf{V}=\underline{\lim _{n \rightarrow \infty}} \sqrt[n]{c_{n}(\mathbf{V})}
$$

Если $f(z)$ - функция сложности, то ее коэффициенты неотрицательны и $M_{f}(r)=f(r)$ для $r>0$. Получаем

СлЕДСТВИЕ 1.1. Пусть V - многообразие ассоииативных алгебр. Тогда

$$
\operatorname{Exp} \mathbf{V}=\varlimsup_{r \rightarrow \infty} \frac{\ln \mathscr{C}(\mathbf{V}, r)}{r}
$$

Предостережем, что замена в лемме $\varlimsup$ нат выражение для Exp V мы записать не можем.

2. Применение функций сложности для $T$-идеалов. В теории свободных групп имеет место следующая формула Шрайера. Пусть $G$ - свободная групша ранга $n$, любая подгруппа $H$ в ней свободна, причем если $|G: H|<\infty$ и $m-$ ранг для $H$, то $m-1=(n-1)|G: H|$.

Известно, что свободная ассоциативная алгебра является $F I$-кольцом, т. е. кольцом, где каждьй односторонний идеал является свободным модулем [9]. Пусть $A=K\left\langle x_{1}, \ldots\right.$ 
$\left.\ldots, x_{k}\right\rangle$ - свободная ассоциативная алгебра ранга $k$ и $J$ - односторонний идеал такой, что $\operatorname{dim} A / J<\infty$. Обозначим через $m$ ранг идеала $J$. Тогда имеет место аналог формулы Шрайера [10]: $m-1=(k-1) \operatorname{dim} A / J$.

Имеет место обобщение этой формулы в терминах рядов. Определим степень элемента $\operatorname{deg} a, a \in A$, как максимум степеней мономов, входящих в его запись. Для подмножества $V \subset A$ будем использовать обозначения $V_{n}=\{a \in V \mid \operatorname{deg} a=n\}$, $V^{n}=\{a \in V \mid \operatorname{deg} a \leqslant n\}, n \geqslant 0$. Пусть теперь свободное порождающее множество $Y$ для ${ }_{A} J$ бесконечно. Рассмотрим ряды Гильберта-Пуанкаре:

$$
\begin{aligned}
\mathscr{H}(Y, t) & =\sum_{n=0}^{\infty}\left|Y_{n}\right| t^{n}, \quad \mathscr{H}(J, t)=\sum_{n=0}^{\infty} \operatorname{dim}\left(J^{n} / J^{n-1}\right) t^{n}, \\
\mathscr{H}(A / J, t) & =\mathscr{H}(A, t)-\mathscr{H}(J, t)=\frac{1}{(1-t)^{k}}-\mathscr{H}(J, t) .
\end{aligned}
$$

Обобщение состоит в следующем [9]:

$$
\mathscr{H}(Y, t)-1=(k t-1) \mathscr{H}(A / J, t) .
$$

Через $A=K\langle X\rangle, X=\left\{x_{i} \mid i \in \mathbb{N}\right\}$, обозначаем свободную ассоциативную алгебру счетного ранга. Далее без дополнительных упоминаний будем использовать равенство $\mathscr{C}_{X}(A, z)=\sum_{n=0}^{\infty} z^{n}=(1-z)^{-1}$. Докажем "экспоненциальную формулу Шрайера" для функций сложности.

Tеорема 2.1. Пусть $A=K\langle X\rangle, X=\left\{x_{i} \mid i \in \mathbb{N}\right\}$. Произвольный $X$-равномерный левый (или правый) идеал $J$ является свободным $A$-модулем ${ }_{A} J=\bigoplus_{y \in Y} A y$, әде множество $Y$ можно выбрать $X$-равномерным, причем

$$
\mathscr{C}(Y, z)-1=(z-1) \mathscr{C}(A / J, z) .
$$

ДокАЗАТЕльство. Известно, что в $A$ каждый односторонний идеал $J$ является свободным [9]. Напомним построение свободного порождающего множества $Y$. Пусть компоненты $Y_{i}, i<n$, уже построены. Тогда в качестве компоненты $Y_{n}$ берем базис дополнения векторного пространства:

$$
J^{n}=\left(A Y^{n-1} \cap J^{n}\right) \oplus\left\langle Y_{n}\right\rangle_{K}, \quad Y^{n-1}=\bigcup_{i<n} Y_{i} .
$$

Согласно "слабому алгоритму" [9] множество $Y=\bigcup_{i \geqslant 0} Y_{i}$ является свободным порождающим для модуля ${ }_{A} J$.

Покажем по индукции, что $Y$ можно построить Х-равномерным. Пусть $Y^{n-1}$ равномерно; так как модуль $A Y^{n-1}$ свободен, то по лемме 1.1 этот модуль также равномерен. Остается заметить, что базис дополнения в (1) также можно выбрать равномерным.

Дополним $J$ до равномерного базиса всей алгебры $A=\bigoplus_{y \in Y} A y \oplus\left\langle b_{i} \mid i \in I\right\rangle_{K}$ и, используя лемму 1.1, вычислим функцию сложности

$$
\begin{aligned}
& \mathscr{C}(A, z)=\mathscr{C}(A, z) \mathscr{C}(Y, z)+\mathscr{C}(I, z), \\
& \mathscr{C}(Y, z)=1+(z-1) \mathscr{C}(A / J, z),
\end{aligned}
$$

что и требовалось доказать. 
Лемма 2.1. Пусть $I, J-T$-идеаль в $A=K\langle X\rangle, X=\left\{x_{i} \mid i \in \mathbb{N}\right\}$. Положим $C_{1}(z)=\mathscr{C}(A / I, z), C_{2}(z)=\mathscr{C}(A / J, z)$. Тогдa

$$
\mathscr{C}(A / I J, z)=C_{1}(z)+C_{2}(z)+(z-1) C_{1}(z) C_{2}(z) .
$$

ДокАЗАТЕльСтво. Согласно теореме 2.1 идеал ${ }_{A} J$ обладает $X$-равномерным свободным порождающим множеством ${ }_{A} J=\bigoplus_{y \in Y} A y$, причем

$$
\mathscr{C}(Y, z)=1+(z-1) \mathscr{C}(A / J, z)=1+(z-1) C_{2}(z) .
$$

Тогда $I \cdot J=\bigoplus_{y \in Y} I y$; вычислим соответствуюшие функции сложности

$$
\begin{aligned}
\mathscr{C}(I J, z) & =\mathscr{C}(I, z) \cdot \mathscr{C}(Y, z), \\
\mathscr{C}(A / I J, z) & =\frac{1}{1-z}-\mathscr{C}(I J, z)=\frac{1}{1-z}-\mathscr{C}(I, z) \mathscr{C}(Y, z) \\
& =\frac{1}{1-z}-\left(\frac{1}{1-z}-C_{1}(z)\right)\left(1+(z-1) C_{2}(z)\right) \\
& =C_{1}(z)+C_{2}(z)+(z-1) C_{1}(z) C_{2}(z) .
\end{aligned}
$$

СлЕДСТвИЕ 2.1. Пусть $I_{i}, i=1, \ldots, c,-T$-идеалы в $A=K\langle X\rangle$, причем $C_{i}=$ $C_{i}(z)=\mathscr{C}\left(A / I_{i}, z\right)$. Тогда имеет место соотношение

$$
\begin{aligned}
\mathscr{C}\left(A /\left(I_{1} \cdots I_{c}\right), z\right)= & C_{1}+\cdots+C_{c}+\cdots \\
& +(z-1)^{j-1} \sum_{i_{1}<\cdots<i_{j}} C_{i_{1}} \cdots C_{i_{j}}+\cdots+(z-1)^{c-1} C_{1} \cdots C_{c} \\
= & \frac{\left(1+(z-1) C_{1}\right) \cdots\left(1+(z-1) C_{c}\right)-1}{z-1} .
\end{aligned}
$$

ДоКАЗАТЕЛЬСТВО по индукции выводится из предыдущей леммы.

Через $N_{c}$ мы обозначим многообразие всех нильпотентных алгебр степени $c$, т. е. совокупность всех алгебр $B$ таких, что $B^{c}=0$. Если $\mathbf{U}, \mathbf{V}$ - многообразия, то $\mathbf{U V}$ обозначает их произведение.

СлЕДСТвИЕ 2.2. Пусть $M$ - многообразие ассочиативных алгебр с функиией сложности $f(z)=\mathscr{C}(M, z)$. Тогда

$$
\mathscr{C}\left(N_{c} M, z\right)=\frac{(1+(z-1) f(z))^{c}-1}{z-1} .
$$

ДокАЗАТЕльСтво. Рассмотрим $T$-идеал $I=T(M) \subset K\langle X\rangle$. Тогда $T\left(N_{c} M\right)=I^{c}$ и по следствию 2.1 вьполняется требуемое равенство. 
СлЕДСТВИЕ 2.3. Пусть $I, J-T$-идеаль в $A=K\langle X\rangle, X=\left\{x_{i} \mid i \in \mathbb{N}\right\}$, задающие многообразия $\mathbf{A}, \mathbf{B}, u a_{n}=c_{n}(\mathbf{A}), b_{n}=c_{n}(\mathbf{B}), n=0,1,2, \ldots$ Тогда

1) имеет место соотношение

$$
c_{n}(A / I J)=a_{n}+b_{n}+n \sum_{i=0}^{n-1}\left(\begin{array}{c}
n-1 \\
i
\end{array}\right) a_{i} b_{n-1-i}-\sum_{i=0}^{n}\left(\begin{array}{c}
n \\
i
\end{array}\right) a_{i} b_{n-i}
$$

2) если $a_{n} \approx \lambda n^{a} \alpha^{n}, b_{n} \approx \mu n^{b} \beta^{n}, \lambda, \mu, a, b, \alpha, \beta \in \mathbb{R}, m o$

$$
c_{n}(A / I J) \approx \lambda \mu \frac{\alpha^{a} \beta^{b}}{(\alpha+\beta)^{a+b+1}} n^{a+b+1}(\alpha+\beta)^{n}
$$

3) имеет место оиенка $\operatorname{Exp}(A / I J) \leqslant \operatorname{Exp} \mathbf{A}+\operatorname{Exp} \mathbf{B}$;

4) $е с л u \operatorname{Exp} \mathbf{A}=\operatorname{Exp} \mathbf{A} u \operatorname{Exp} \mathbf{B}=\operatorname{Exp} \mathbf{B}, m o$

$$
\operatorname{Exp}(A / I J)=\underline{\operatorname{Exp}}(A / I J)=\operatorname{Exp} \mathbf{A}+\operatorname{Exp} \mathbf{B} .
$$

ДокАЗАТЕЛЬСТво. 1) Перемножаем производящие функции (2) и приравниваем коэффициенты в левой и правой части.

2) Рассмотрим вспомогательную последовательность

$$
d_{n}=\sum_{i=0}^{n}\left(\begin{array}{c}
n \\
i
\end{array}\right) a_{i} b_{n-i}, \quad n=0,1,2, \ldots
$$

Известно [11], [6], что при заданных условиях имеет место асимптотика

$$
d_{n} \approx \lambda \mu \frac{\alpha^{a} \beta^{b}}{(\alpha+\beta)^{a+b}} n^{a+b}(\alpha+\beta)^{n}
$$

Тогда $c_{n}(A / I J)=a_{n}+b_{n}+n d_{n-1}-d_{n}, n \in \mathbb{N}$, и несложные вычисления дают требуемьй результат.

3) Рост функции сложности $\mathscr{C}(A / I J, z)$ определяется последним слагаемым в $(2)$. Используя следствие 1.1, получаем

$\operatorname{Exp}(A / I J)=\varlimsup_{r \rightarrow \infty} \frac{\ln \mathscr{C}(A / I J, r)}{r}=\varlimsup_{r \rightarrow \infty} \frac{\ln ((r-1) \mathscr{C}(\mathbf{A}, r) \mathscr{C}(\mathbf{B}, r))}{r} \leqslant \operatorname{Exp} \mathbf{A}+\operatorname{Exp} \mathbf{B}$.

4) Требуемое соотношение выводится несложньми рассуждениями из 1), см., например, [11], [6].

ЗАмЕчАниЕ 1. В нулевой характеристике утверждение следствия 2.3 известно [11], [6], оно основано на явной формуле для характера $\chi_{n}(A / I J)$.

ЗАмЕчАниЕ 2. Формула (2) является аналогом следуюшей хорошо известной формулы [12]. Пусть $I, J-T$-идеалы в конечно-порожденной свободной ассоциативной алгебре $A=K\left\langle x_{1}, \ldots, x_{k}\right\rangle$. Тогда для рядов Гильберта-Пуанкаре выполнено соотношение

$$
\mathscr{H}(A / I J, t)=\mathscr{H}(A / I, t)+\mathscr{H}(A / J, t)+(k t-1) \mathscr{H}(A / I, t) \mathscr{H}(A / J, t)
$$




\section{3. Производящие функции для алгебры верхнетреугольных матриц.}

ТеоРема 3.1. Пусть $\mathbf{U}_{c}$ - многообразие ассочиативных алгебр, порожденное алгеброй верхнетреугольных матрии размера $c \times c$. Тогда функиия сложности этого многообразия равна

$$
\mathscr{C}\left(\mathbf{U}_{c}, z\right)=\frac{(1+(z-1) \exp z)^{c}-1}{z-1} .
$$

ДокАЗАТЕЛЬСТво. Многообразие $\mathbf{U}_{c}$ над полем нулевой характеристики задается одним тождеством [13]

$$
\left[X_{1}, X_{2}\right] \cdot\left[X_{3}, X_{4}\right] \cdots\left[X_{2 c-1}, X_{2 c}\right] \equiv 0
$$

Над произвольньм полем $K$ имеет место соотношение $T\left(\mathbf{U}_{c}\right)=(T(K))^{c}$, где $T(K)-$ идеал тождеств многообразия $\operatorname{var} K$. В случае бесконечного поля он порождается тождеством $[X, Y] \equiv 0$; если же $|K|=q$, то добавляется тождество $X^{q}-X \equiv 0[14]$. В любом случае $P_{n}(K)=\left\langle x_{1} x_{2} \cdots x_{n}\right\rangle_{K}, c_{n}(K)=1, n=0,1,2, \ldots$, и, следовательно, $\mathscr{C}(\operatorname{var} K, z)=\sum_{n=0}^{\infty} z^{n} / n !=\exp (z)$. Осталось применить следствие 2.2 к $\mathbf{U}_{c}=$ $\mathbb{N}_{c} \operatorname{var} K$.

В пространстве многочленов вместо базиса $n^{i}, i=0,1,2, \ldots$, рассмотрим следующий базис $(n)_{i}, i=0,1,2, \ldots$ :

$$
(n)_{0}=1, \quad(n)_{i}=n(n-1) \cdots(n-i+1), \quad i=1,2, \ldots .
$$

Назовем функции вида $f(n)=\lambda^{n} n^{a}, \lambda, a \in \mathbb{R}, n=0,1,2, \ldots$, и их линейные комбинации квазиполиномами.

СледСтвиЕ 3.1. Функиия роста коразмерностей для алгебры верхнетреугольных матрич является следующим квазиполиномом:

$$
c_{n}\left(\mathbf{U}_{c}\right)=\sum_{s=1}^{c} s^{n} \sum_{i=0}^{s-1} \lambda_{s i}(n)_{i}, \quad n=0,1,2, \ldots,
$$

әде коэффициенты равны

$$
\lambda_{s i}=\left(\begin{array}{c}
c \\
s
\end{array}\right)\left(\begin{array}{c}
s-1 \\
i
\end{array}\right) s^{-i}(-1)^{s-i-1} .
$$

ДокАЗАТЕЛЬСТво. Представим функцию сложности в виде

$$
\mathscr{C}\left(\mathbf{U}_{c}, z\right)=\sum_{s=1}^{c}\left(\begin{array}{l}
c \\
s
\end{array}\right)(z-1)^{s-1} \exp (s z)
$$


и разложим

$$
\begin{aligned}
(z-1)^{s-1} \exp (s z) & =\sum_{i=0}^{s-1}\left(\begin{array}{c}
s-1 \\
i
\end{array}\right) z^{i}(-1)^{s-1-i} \sum_{n=0}^{\infty} \frac{z^{n} s^{n}}{n !}=(n+i=m) \\
& =\sum_{m=0}^{\infty} \frac{z^{m}}{m !} \sum_{i=0}^{\min \{m, s-1\}}\left(\begin{array}{c}
s-1 \\
i
\end{array}\right)(-1)^{s-1-i} s^{m-i} \frac{m !}{(m-i) !} \\
& =\sum_{m=0}^{\infty} \frac{z^{m}}{m !} s^{m}\left(\sum_{i=0}^{s-1}\left(\begin{array}{c}
s-1 \\
i
\end{array}\right)(-1)^{s-1-i} s^{-i} \cdot(m)_{i}\right) .
\end{aligned}
$$

Подставляя это в (4), имеем

$$
\mathscr{C}\left(\mathbf{U}_{c}, z\right)=\sum_{m=0}^{\infty} \frac{z^{m}}{m !}\left(\sum_{s=1}^{c} s^{m} \sum_{i=0}^{s-1}\left(\begin{array}{l}
c \\
s
\end{array}\right)\left(\begin{array}{c}
s-1 \\
i
\end{array}\right)(-1)^{s-1-i} s^{-i} \cdot(m)_{i}\right)
$$

Мы использовали, что $(m)_{i}=0$ при $i \geqslant m+1$. Коэффициенты в скобках и есть искомые $c_{m}\left(\mathbf{U}_{c}\right)$.

Рассмотрим также обычную производящую функцию для последовательности коразмерностей $\mathscr{H}\left(c_{n}(\mathbf{V}), t\right)=\sum_{n=0}^{\infty} c_{n}(\mathbf{V}) t^{n}$.

СлЕДСТВИЕ 3.2. Объчная производящая функиия $\mathscr{H}\left(c_{n}\left(\mathbf{U}_{c}\right), t\right)$ рациональна и равна

$$
\mathscr{H}\left(c_{n}\left(\mathbf{U}_{c}\right), t\right)=\sum_{s=1}^{c} \sum_{k=0}^{s-1} \mu_{s k} \frac{t^{k}}{(1-s t)^{k+1}}
$$

где коэффичиенты задаются формулой

$$
\mu_{s k}=\left(\begin{array}{l}
c \\
s
\end{array}\right)(s-1)_{k}(-1)^{s-1-k}
$$

ДОКАЗАТЕЛЬСТво. Пусть

$$
E(z)=\sum_{n=0}^{\infty} \frac{a_{n}}{n !} z^{n}
$$

- целая функция экспоненциального типа, причем $\sigma=\varlimsup_{n \rightarrow \infty} \sqrt[n]{\left|a_{n}\right|}$. Рассмотрим для последовательности $a_{n}, n=0,1,2, \ldots$, также обычную производящую функцию $H(t)=\sum_{n=0}^{\infty} a_{n} t^{n}$. Тогда $H(t)$ имеет радиус сходимости $|t|<1 / \sigma$. Имеет место формальное преобразование [15]:

$$
\begin{aligned}
\mathscr{B}(E(z))(t) & =\int_{0}^{\infty} e^{-x} E(t x) d x=\int_{0}^{\infty} e^{-x} \sum_{n=0}^{\infty} \frac{a_{n}}{n !} t^{n} x^{n} d x \\
& =\sum_{n=0}^{\infty} \frac{a_{n}}{n !} t^{n} \int_{0}^{\infty} e^{-x} x^{n} d x=\sum_{n=0}^{\infty} a_{n} t^{n}=H(t), \quad|t|<\frac{1}{\sigma} .
\end{aligned}
$$


Законность преобразований проверяется аналогично [16, п. 519]. Для функции $E_{k s}(z)=$ $z^{k} \exp (s z)$ получаем

$$
\begin{aligned}
\mathscr{B}\left(E_{k s}(z)\right)(t) & =\int_{0}^{\infty} e^{-x}(t x)^{k} e^{s t x} d x=t^{k} \int_{0}^{\infty} x^{k} e^{-x(1-s t)} d x \\
& =\frac{t^{k}}{(1-s t)^{k+1}} \int_{0}^{\infty} y^{k} e^{-y} d y=\frac{t^{k} k !}{(1-s t)^{k+1}}, \quad|t|<\frac{1}{s} .
\end{aligned}
$$

Используя (4), имеем

$$
\begin{aligned}
\mathscr{C}\left(\mathbf{U}_{c}, z\right) & =\sum_{s=1}^{c}\left(\begin{array}{l}
c \\
s
\end{array}\right) \sum_{k=0}^{s-1}\left(\begin{array}{c}
s-1 \\
k
\end{array}\right) z^{k}(-1)^{s-1-k} \exp (s z) \\
& =\sum_{s=1}^{c} \sum_{k=0}^{s-1}\left(\begin{array}{c}
c \\
s
\end{array}\right)\left(\begin{array}{c}
s-1 \\
k
\end{array}\right)(-1)^{s-1-k} E_{k s}(z), \\
\mathscr{B}\left(\mathscr{C}\left(\mathbf{U}_{c}, z\right)\right)(t) & =\sum_{s=1}^{c} \sum_{k=0}^{s-1}\left(\begin{array}{c}
c \\
s
\end{array}\right)\left(\begin{array}{c}
s-1 \\
k
\end{array}\right) k !(-1)^{s-1-k} \frac{t^{k}}{(1-s t)^{k+1}},
\end{aligned}
$$

что и дает требуемую формулу.

В [17] доказано, что для любого подмногообразия $\mathbf{V} \subset \mathbf{U}_{c}$ в случае бесконечного поля ряд Гильберта-Пуанкаре $\mathscr{H}(F(\mathbf{V}, k), t)$ свободной алгебры конечного ранга рационален. Получим для $\mathbf{U}_{c}$ точную формулу.

Лемма 3.1. Пусть $\mathscr{H}\left(F\left(\mathbf{U}_{c}, k\right), t\right)=\sum_{n=0}^{\infty} a_{n} t^{n}-$ ряд Гильберта-Пуанкаре свободной алгебры ранга $k$ над бесконечныцм полем. Тогда

1) имеет место равенство

$$
\mathscr{H}\left(F\left(\mathbf{U}_{c}, k\right), t\right)=\frac{1}{k t-1}\left(\left(1+\frac{k t-1}{(1-t)^{k}}\right)^{c}-1\right) \approx \frac{(k-1)^{c-1}}{(1-t)^{k c}}, \quad t \rightarrow 1-0
$$

2) функция $a_{n}$ является полиномом, причем $a_{n} \approx(k-1)^{c-1} n^{k c-1} /(k c-1)$ !.

ДокаЗАТЕльство. В этом случае $T\left(\mathbf{U}_{c}\right)=(T(K))^{c}$ и $\mathscr{H}(A / I, t)=(1-t)^{-k}$, где $A=K\left\langle x_{1}, \ldots, x_{k}\right\rangle, I=T(K)$. Далее применяем (3) к $I^{c}$ и по индукции доказываем искомую формулу. Второе утверждение следует из свойств рациональных функций.

ЗАмЕчание 3. Очевидна аналогия с теоремой 3.1. В отличие от [17], нами для $\mathbf{U}_{c}$ найдена точная формула ряда и установлено, что $a_{n}$ является полиномом.

4. Собственные тождества. Следующие утверждения о собственных тождествах известны для нулевой характеристики [18]-[20], хотя использование характеров и нулевой характеристики является излишним. Покажем, что в терминах функций сложности и характеристик их роста следуюшие факты становятся прозрачными.

В свободной алгебре счетного ранга $F(\mathbf{V}, X)$ ассоциативного многообразия $\mathbf{V}$ рассмотрим подпространство, натянутое на произведения коммутаторов и единицу. Пусть 
$P_{n}^{p}(\mathbf{V})$ - пересечение этого подпространства с пространством полилинейных элементов $P_{n}\left(\left\{x_{1}, \ldots, x_{n}\right\}, \mathbf{V}\right)$. Возникают функция роста собственных тождеств, соответствующие ей функция сложности и экспоненты:

$$
\begin{aligned}
c_{n}^{p}(\mathbf{V}) & =\operatorname{dim} P_{n}^{p}(\mathbf{V}), \quad n=0,1,2, \ldots, & \mathscr{C}^{p}(\mathbf{V}, z) & =\sum_{n=0}^{\infty} \frac{c_{n}^{p}(\mathbf{V})}{n !} z^{n} \\
\operatorname{Exp}^{p} \mathbf{V} & =\varlimsup_{n \rightarrow \infty} \sqrt[n]{c_{n}^{p}(\mathbf{V})}, & \underline{\operatorname{Exp}}^{p} \mathbf{V} & =\underline{\lim }_{n \rightarrow \infty} \sqrt[n]{c_{n}^{p}(\mathbf{V})}
\end{aligned}
$$

Также в свободной алгебре конечного ранга $F(\mathbf{V}, k)$ рассмотрим подпространство $F^{p}(\mathbf{V}, k)$, натянутое на произведения коммутаторов и единицу. Возникает соответствующая функция $\mathscr{H}_{k}^{p}(\mathbf{V}, t)$.

ЛЕмма 4.1 [18]. Пусть $\mathbf{V}=\operatorname{var} M$, әде $M-$ ассоциативная алгебра с единицей. Тогда

1) $\mathscr{C}(\mathbf{V}, z)=\exp (z) \mathscr{C}^{p}(\mathbf{V}, z)$

2) $\mathscr{H}_{k}(\mathbf{V}, t)=\left(1 /(1-t)^{k}\right) \mathscr{H}_{k}^{p}(\mathbf{V}, t)$, если $\mathbf{V}$ полиоднородно (в частности, если поле бесконечно).

ДокАЗАТЕЛЬСТво. Пусть алгебра $F(\mathbf{V}, k)$ порождена элементами $x_{1}, \ldots, x_{k}$. Используя преобразования $a x_{i} x_{j} b=a x_{j} x_{i} b+a\left[x_{i} x_{j}\right] b$, мы вытаскиваем отдельные буквы в начало мономов упорядоченньм образом, оставляя коммутаторы справа:

$$
x_{i_{1}} \cdots x_{i_{s}} \cdots\left[x_{j_{1}}, \ldots\right] \cdots\left[x_{j_{m}}, \ldots\right], \quad x_{i_{1}} \leqslant \cdots \leqslant x_{i_{s}} .
$$

Рассмотрим линейное отображение

$$
\varphi: K\left[x_{1}, \ldots, x_{k}\right] \otimes F^{p}(\mathbf{V}, k) \rightarrow F(\mathbf{V}, k),
$$

задаваемое формулой

$$
\varphi\left(x_{1}^{m_{1}} \cdots x_{k}^{m_{k}} \otimes w\right)=x_{1}^{m_{1}} \cdots x_{k}^{m_{k}} w, \quad w \in F^{p}(\mathbf{V}, k) .
$$

Докажем, что $\varphi$ является изоморфизмом векторных пространств

$$
F(\mathbf{V}, k) \cong K\left[x_{1}, \ldots, x_{k}\right] \otimes F^{p}(\mathbf{V}, k)
$$

В силу условия рассмотрим однородный базис $\left\{w_{j} \mid j \in J\right\}$ для $F^{p}(\mathbf{V}, k)$ и предположим, что имеет место зависимость $\sum_{\alpha, j} \mu_{\alpha, j} x_{1}^{\alpha_{1}} \cdots x_{k}^{\alpha_{k}} w_{j}=0, \mu_{\alpha, j} \in K$. Пусть $w_{s}$ элемент минимальной степени, входящий в сумму с ненулевым коэффищиентом. При подстановке $x_{i}=x_{i}+1, i=1, \ldots, k$, элементы $w_{j}$ не меняются, так как состоят из коммутаторов. Рассматривая однородную компоненту степени $m=\operatorname{deg} w_{s}$, получаем $\sum_{\alpha, i} \mu_{\alpha, i} w_{i}=0$, где $\operatorname{deg} w_{i}=m$, что противоречит выбору $w_{j}$ как базиса для $F^{p}(\mathbf{V}, k)$.

Пусть $P(B)$ обозначает линейную оболочку всех полилинейных компонент степеней $n=0,1,2, \ldots$ в $B$. Аналогично доказывается, что для свободной алгебры счетного ранга имеет место изоморфизм полилинейных компонент:

$$
P(F(X, \mathbf{V})) \cong P\left(K[X] \otimes F^{p}(X, \mathbf{V})\right), \quad X=\left\{x_{i} \mid i \in \mathbb{N}\right\}
$$

Теперь искомые соотношения вытекают из (5), (6), свойств обычных и экспоненциальных производящих функций (лемма 1.1) и соотношений

$$
\mathscr{H}\left(K\left[x_{1}, \ldots, x_{k}\right], t\right)=(1-t)^{-k}, \quad \mathscr{C}(K[X], z)=\exp (z)
$$

для $X=\left\{x_{i} \mid i \in \mathbb{N}\right\}$. 
СлЕДСТВИЕ 4.1 [20]. Пусть $\mathbf{V}=\operatorname{var} M$, әде $M-$ ассоциативная алгебра с единичей. Тогда $\operatorname{Exp}(\mathbf{V})=\operatorname{Exp}^{p}(\mathbf{V})+1$.

ДоКАЗАТЕЛЬСТВо. По следствию 1.1 и его аналогу для $\operatorname{Exp}^{p}(\mathbf{V})$ получаем

$$
\operatorname{Exp} \mathbf{V}=\varlimsup_{r \rightarrow \infty} \frac{\ln \mathscr{C}(\mathbf{V}, r)}{r}=\varlimsup_{r \rightarrow \infty} \frac{\ln \left(\exp (r) \mathscr{C}^{p}(\mathbf{V}, r)\right)}{r}=\operatorname{Exp}^{p}(\mathbf{V})+1
$$

\section{СПИСОК ЦИТИРОВАННОЙ ЛИТЕРАТУРЫ}

[1] Regev A. Existence of identities in $A \otimes B / /$ Israel J. Math. 1972. V. 11. P. 131-152.

[2] Размыслов Ю. П. Тождества алгебр и их представлений. М.: Наука, 1989.

[3] Воличенко И. Б. О многообразии алгебр Ли $\mathbf{A} \mathbb{N}_{2}$ над полем характеристики нуль // Докл. АН БССР. 1981. Т. 25. № 12. С. 1063-1066.

[4] Петроградский В. М. О типах сверхэкспоненциального роста тождеств в PI-алгебрах Ли // Фундамент. и прикл. матем. 1995. Т. 1. № 4. С. 989-1007.

[5] Петроградский В. М. Рост полинильпотентных многообразий алгебр Ли и быстро растущие целые функции // Матем. сб. 1997. Т. 188. №6. С. 119-138.

[6] Regev A. Asymptotics of codimensions of some P.I.-algebras // Trends in Ring Theory (Miskolc, 1996). CMS Conf. Proc. V. 22. Providence, R.I.: Amer. Math. Soc., 1998. P. 159-172.

[7] Гульден Я., Джексон Д. Перечислительная комбинаторика. М.: Наука, 1990.

[8] Леонтьев А. Ф. Целые функции. Ряды экспонент. М.: Наука, 1983.

[9] Кон П. Свободные кольца и их связи. М.: Мир, 1975.

[10] Lewin J. Free modules over free algebras and free group algebras: Thev Schreier technique // Trans. Amer. Math. Soc. 1969. V. 145. P. 455-465.

[11] Berele A. Regev A. Codimensions of products and of intersections of verbally prime $T$-ideals // Israel J. Math. 1998. V. 103. P. 17-28.

[12] Halpin P. Some Poincaré series related to identities of $(2 \times 2)$ matrices // Pacific J. Math. 1983. V. 107. № 1. P. 107-115.

[13] Мальцев Ю. Н. Базис тождеств алгебры верхнетреугольных матриц // Алгебра и логика. 1971. Т. 10. № 4. С. 393-400.

[14] Сидеров П. Н. Базис тождеств алгебры треугольных матриц над произвольным полем // Плиска. Български Математически Студии. 1981. Т. 2. С. 143-152.

[15] Риордан Дж. Введение в комбинаторный анализ. М.: ИЛ, 1963.

[16] Фихтенгольц Г. М. Курс дифференциального и интегрального исчисления. Т. 1-3. М.: Наука, 1969.

[17] Drensky V. On the Hilbert series of relatively free algebras // Comm. Algebra. 1984. V. 12. № 19. P. 2335-2347.

[18] Drensky V. Codimensions of $T$-ideals and Hilbert series of relatively free algebras // J. Algebra. 1984. V. 91. № 1. P. 1-17.

[19] Drensky V., Regev A. Exact asymptotic behaviour of the codimensions of some P.I.-algebras // Israel J. Math. 1996. V. 96. part A. P. 231-242.

[20] Bahturin Yu., Mishchenko S., Regev A. On the Lie and associative codimension growth // Comm. Algebra. (to appear).

Ульяновский государственный университет

Поступило

E-mail: vmp@mmf.univ.simbirsk.su

07.05.1999 